Национальный медицинский исследовательский центр эндокринологии, Москва, Россия

ОБОСНОВАНИЕ. Синдром Клайнфельтера (СК) - распространенное генетическое заболевание, характеризующееся гипергонадотропным гипогонадизмом. Высокий риск развития метаболических нарушений у пациентов с СК может объясняться наличием андрогенного дефицита, что приводит к снижению количества тощей массы и увеличению содержания жировой ткани в организме. Скорость основного обмена (ОО) определяется количеством тощей массы, а его снижение может способствовать набору веса и прогрессированию метаболических нарушений при СК.

ЦЕЛЬ. Анализ композиционного состава тела, оценка ОО и метаболического профиля у подростков с СК.

МАТЕРИАЛЫ И МЕТОДЫ. В исследование были включены 28 подростков с СК, сопоставимых по возрасту и стадии полового развития. Все пациенты были разделены на две группы в зависимости от наличия клинико-лабораторных признаков гипогонадизма. Пациентам были проведены оценка метаболического профиля, биоимпедансометрия для анализа композиционного состава тела и непрямая респираторная калориметрия для оценки ОО.

РЕЗУЛЬТАТЫ. У 20 (71,4\%) подростков определились нормальные показатели композиционного состава тела, избыток жировой массы - у 6 (21,4\%) пациентов, дефицит жировой массы - в 7,2\% случаев. Среди 6 пациентов с избытком жировой массы у трех отмечалось ожирение или избыточная масса тела, остальные имели нормальный SDS индекса массы тела (ИМТ). У 64,3\% подростков выявлены нормальные показатели энергообмена в покое, у 6 (21,4\%) - снижение скорости ОО, у 4 (14,3\%) - повышение скорости ОО. При сравнительном анализе групп пациентов не выявлено статистически значимых различий по показателю ОО с поправкой на тощую массу $(\mathrm{OO} / \mathrm{TM})(\mathrm{p}=0,36)$. Инсулинорезистентность выявлена у 11 пациентов (39,3\%), частота дислипидемии составила 3,6\%. По уровням гликемии натощак и показателям липидного профиля исследуемые группы значимо не различались.

ЗАКЛЮЧЕНИЕ. У большинства подростков с СК определяются нормальные показатели композиционного состава тела и основного обмена, а также низкая частота метаболических нарушений, независимо от уровня общего тестостерона в крови. У части пациентов с нормальным SDS ИМТ выявляется избыток жировой массы. Отсутствие взаимосвязи уровня тестостерона с интенсивностью ОО может свидетельствовать о незначительном влиянии андрогенного дефицита на энергообмен в покое у подростков с СК.

КЛЮЧЕВЫЕ СЛОВА: СИндром Клайнфельтера, основной обмен, композичионный состав тела.

\title{
EVALUATION OF BODY COMPOSITION, RESTING METABOLIC RATE AND FREQUENCY OF METABOLIC DISORDERS IN ADOLESCENTS WITH KLINEFELTER SYNDROME
}

\author{
(c) Daria A. Bespalyuk*, Pavel L. Okorokov, Igor S. Chugunov
}

Endocrinology Research Centre, Moscow, Russia

BACKGROUND: Kleinfelter syndrome (KS) is a common genetic disease characterized by hypergonadotropic hypogonadism. The high risk of developing of metabolic disorders in patients with KS is be explained by the presence of androgen deficiency, which leads to a decrease in the amount of lean soft mass and an increase of the adipose tissue content. The basal metabolic rate (BMR) is determined by the amount of lean soft mass, and its reduction can contribute to weight gain and the progression of metabolic disorders in KS.

AIM: Body composition, assessment of basal metabolism and metabolic profile in adolescents with KS.

MATERIALS AND METHODS: The study included 28 adolescents with KS, comparable in age and stage of sexual maturation. All patients were divided into two groups depending on the presence of clinical laboratory signs of hypogonadism. Patients passed through the evaluation of metabolic profile, bioelectrical impedance analysis for the body composition and chamber-based indirect calorimetry for the evaluation of BMR.

RESULTS: Normal indicants of body composition were determined in 20 (71.4\%) adolescents, excess of adipose mass - in $6(21.4 \%)$ patients, and deficit of adipose mass - in 7.2\% of cases. Among 6 patients with excess of adipose mass, three were obese or overweight, while the rest had normal SDS body mass index (BMI). $64.3 \%$ of adolescents showed normal indicants 
of energy exchange at rest, 6 (21.4\%) - a decrease of BMR, 4 (14.3\%) - an increase of BMR. A comparative analysis of patient groups did not reveal statistically significant differences in BMR adjusted to a lean soft mass (OO/TM) ( $p=0.36)$. Insulin resistance was detected in 11 patients (39.3\%), and the incidence of dyslipidemia was 3.6\%. The groups did not significantly differ in terms of glycemia levels in the fasted state and lipid profile indicants.

CONCLUSION: Most adolescents with KS have normal indicants of body composition and basal metabolism, as well as a low frequency of metabolic disorders, regardless of the level of total testosterone in blood. In some patients with normal SDS BMI, excess of adipose mass is detected. The lack of correlation between the level of testosterone and the intensity of BMR may indicate a slight effect of androgen deficiency on energy exchange at rest in adolescents with KS.

KEYWORDS: Kleinfelter syndrome, basal metabolism, body composition.

\section{ОБОСНОВАНИЕ}

Синдром Клайнфельтера (СК) является распространенной хромосомной аномалией с наличием в кариотипе одной или нескольких дополнительных Х-хромосом. Данное заболевание характеризуется клинико-лабораторными проявлениями гипергонадотропного гипогонадизма и высокой частотой метаболических нарушений [1]. Примерно 50\% взрослых пациентов с СК имеют ожирение с преимущественным распределением жира в абдоминальной области [2]. В ряде работ было продемонстрировано увеличение процентного содержания жировой ткани у детей и подростков с СК еще до появления признаков гипогонадизма и даже при нормальном индексе массы тела (ИМТ) [3, 4], однако топографические особенности распределения жировой ткани при этом не изучались. Риск развития метаболического синдрома у взрослых пациентов с СК по сравнению с общей популяцией выше в 4-5 раз [5]. Так, частота инсулинорезистентности достигает 30\% [6], а сахарного диабета 2 типа - 10\% [2]. Известно, что нарушения углеводного и жирового обмена могут развиваться у пациентов с другими формами гипогонадизма, а также на фоне возрастного андрогенного дефицита у здоровых мужчин, указывая на важную роль тестостерона в процессах метаболизма [7]. При дефиците андрогенов у взрослых пациентов с СК отмечается уменьшение количества тощей массы в организме [5], вносящей основной вклад в вариабельность энергетического обмена в покое [8]. Снижение интенсивности основного обмена может предрасполагать к развитию и прогрессии конституционально-экзогенного ожирения [9]. Таким образом, можно предположить, что снижение уровня андрогенов при СК ассоциировано со снижением интенсивности основного обмена. Изучение энергообмена в покое при СК как во взрослой, так и в детской популяции ранее не проводилось.

\section{ЦЕЛЬ}

Оценить параметры композиционного состава тела, уровня основного обмена и частоты метаболических нарушений у подростков с СК в зависимости от наличия клинических и лабораторных признаков гипогонадизма.

\section{МЕТОДЫ}

\section{Дизайн исследования}

В обсервационное одноцентровое одномоментное выборочное исследование были включены 28 подростков с СК.
Критерии соответствия

Критериями включения в исследование являлись подтвержденный методом кариотипирования диагноз СК и достижение стадии полового развития по Таннеру 3-4.

Критерии исключения: тяжелые психические расстройства, отказ пациента и/или его родителей от участия в исследовании.

Условия проведения

Обследование подростков проводилось на базе НИИ детской эндокринологии ФГБУ «НМИЦ эндокринологии» Минздрава России.

Продолжительность исследования

Набор пациентов проводился в период с декабря 2017 г. по ноябрь 2019 г.

Описание медицинского вмешательства

Всем пациентам было проведено клинико-функциональное обследование, включавшее: антропометрические измерения (рост, вес, расчет ИМТ и SDS («standard deviation score» - стандартное отклонение) ИМТ, определение SDS верхнего и нижнего сегментов тела), рентгенографию кистей рук с целью определения «костного возраста», определение содержания в сыворотке крови общего тестостерона, липидного профиля (триглицеридов, общего холестерина, липопротеидов низкой плотности (ЛПНП), липопротеидов высокой плотности (ЛПВП)), аланинаминотрансферазы (АЛТ), аспартатаминотрансферазы (АСТ), глюкозы, иммунореактивного инсулина (ИРИ) и расчет индекса НОМА.

\section{Основной исход исследования}

Основными конечными точками исследования являлись показатели количества жировой и тощей массы по данным биоимпедансометрии и основного обмена (ОО), исследованные методом непрямой респираторной калориметрии.

\section{Анализ в подгруппах}

Пациенты, сопоставимые по возрасту и стадии полового развития по Таннеру, были разделены на 2 группы. В 1-ю группу были включены 11 пациентов, имевших на момент обследования клинические и лабораторные признаки гипогонадизма: диспропорцию сегментов тела (разница между SDS верхнего сегмента и SDS нижнего сегмента тела $> \pm 2$ ), уровень тестостерона сыворотки крови <9 нмоль/л; 2-я группа включала 17 пациентов, не имевших на момент обследования клинических и лабораторных признаков гипогонадизма. 
Методы регистрации исходов

Измерение роста, величины верхнего и нижнего сегментов тела проводилось на ростомере SECA с точностью до 0,1 см, масса тела была измерена с помощью весов SECA с точностью до 0,01 кг. Расчет показателей SDS роста, ИМТ, сегментов тела производился в компьютерном приложении Auxology 1,0 b17 (Pfizer, США). Наличие избыточной массы тела и ожирения диагностировалось согласно критериям ВОЗ. Стадия полового развития оценивалась по классификации Таннера. Определение в плазме крови уровня глюкозы, в сыворотке крови уровня триглицеридов (ТГ), общего холестерина, холестерина ЛПНП, ЛПВП, АЛТ, АСТ проводилось на биохимическом анализаторе Architect plusC4000 (Abbott Diagnostics, США). Содержание в сыворотке крови общего тестостерона исследовалось методом усиленной хемилюминесценции с помощью автоматического иммунохимического анализатора Vitros 3600 (Ortho Clinical Diagnostics, Johnson\&Johnson, США). Определение уровня ИРИ проводилось методом усиленной хемилюминесценции на анализаторе COBAS 6000 фирмы RocheDiagnostics (Швейцария). Состояние углеводного обмена оценивалось по результатам орального глюкозотолерантного теста (ОГТТ) с нагрузкой глюкозой (из расчета 1,75 г на кг веса, но не более 75 г) согласно критериям ВОЗ (1999). Для оценки инсулинорезистентности (ИР) использовался индекс HOMA-IR (Homeostasis Model Assessment of Insulin Resistance), рассчитанный по формуле: (ИРИ (базальный уровень) $\times$ Гл (базальный уровень))/22,5, где ИРИ - иммунореактивный инсулин, мкЕд/мл; Гл - глюкоза, ммоль/л. ИР диагностировалась при значении индекса НОМА >3,2.

«Костный возраст» оценивался по данным рентгенографии кистей рук с лучезапястными суставами и определялся в соответствии с общепринятой методикой по атласу W. Greulich, S.I. Pyle. Radiograph Atlas of Skeletal Development of the Hand and Wrist. Stanford University Press, Stanford, California (1959). Оценка композиционного состава тела проведена методом биоимпедансного анализа на аппарате Tanita BC-418MA. Нормативные значения количества жировой ткани для данного анализатора представлены в работе McCarthy и соавт. [10].

Величина ОО определялась методом непрямой респираторной калориметрии на метаболографе Quark RMR (Cosmed, Италия). Исследование выполнялось в утренние часы, строго натощак с периодом голодания не менее 7 ч, в положении пациента лежа в спокойной обстановке при температуре в помещении $22-26^{\circ} \mathrm{C}$, продолжительностью 15-20 минут. Расчетные значения ОО определены по формуле Харриса-Бенедикта. При проведении статистического анализа ОО был представлен с поправкой на тощую массу, для чего был использован показатель ОО/ТМ [8].

\section{Этическая экспертиза}

Проведение данного исследования одобрено локальным этическим комитетом по этике ФГБУ «НМИЦ эндокринологии» МЗ РФ (протокол № 20 от 08.11.2017 г.). Все пациенты и их законные представители подписывали добровольное информированное согласие на участие в исследовании.

\section{Статистический анализ}

Размер выборки предварительно не рассчитывался. Обработка полученных данных проводилась с помощью программы Microsoft Excel и пакета прикладных программ Statistica (StatSoft Inc., США, версия 12.0). Учитывая, что исследуемые параметры не имели нормального распределения, в работе произведен расчет медиан с указанием нижнего и верхнего квартиля (Ме [QH; QB]). Статистическая обработка полученных результатов проводилась с помощью непараметрических критериев статистического анализа. Корреляционный анализ переменных проводился с помощью ранговой корреляции по Спирмену. Сравнение двух независимых групп проводилось с помощью U-критерия Манна-Уитни. Уровень статистической значимости различий принимался при $\mathrm{p}<0,05$.

\section{РЕЗУЛЬТАТЫ}

Объекты (участники) исследования

Объектами исследования являлись 28 подростков с СК, сопоставимых по возрасту и стадии полового развития по Таннеру, разделенных на 2 группы в зависимости от наличия симптомов гипогонадизма на момент обследования. Характеристика клинико-лабораторных показателей пациентов обеих групп представлена в таблице 1.

\section{Основные результаты исследования}

Ожирение выявлено у 2 подростков с СК (7,1\%), избыточная масса тела - у 1 подростка (3,6 \%), нормальный ИМТ - у 25 подростков (89,3\%).

При проведении биоимпедансного анализа у 71,4\% подростков с CK $(n=20)$ выявлен нормальный композиционный состав тела. Избыток жировой ткани определен у $21,4 \%$ пациентов $(n=6)$, дефицит жировой ткани - в 7,2\% случаев $(\mathrm{n}=2)$.

Таблица 1. Сравнительная характеристика пациентов обеих групп

\begin{tabular}{lccc}
\hline \multicolumn{1}{c}{ Группа } & $\mathbf{1}$ & $\mathbf{2}$ & $\mathbf{p}$ \\
\hline $\mathrm{n}$ & 11 & 17 & 0,57 \\
Возраст, годы & $15,8[15,3 ; 17,0]$ & $16,04[14,6 ; 16,7]$ & 0,65 \\
Костный возраст, годы & $16,5[14,5 ; 17,0]$ & $16,5[15,0 ; 17,0]$ & 0,3 \\
Стадия по Таннеру & $3[3 ; 4]$ & $4[3 ; 4]$ & 0,003 \\
T общий, нмоль/л & $8,7[4,0 ; 8,9]$ & $11,4[9,2 ; 12,6]$ & 0,37 \\
SDS ИМт & $0,3[-0,5 ; 0,4]$ & $-0,2[-1,3 ; 0,3]$ & \\
\hline
\end{tabular}

Примечание. Данные представлены в виде: Мe [QH; QB]. Уровень статистической значимости различий принимался при р<0,05. 
При оценке процентного содержания жировой ткани в организме в зависимости от наличия гипогонадизма не выявлено значимых различий между исследуемыми группами (16,3 vs 17\%; р>0,05). В группе с клиническими признаками гипогонадизма $(n=11)$ у двоих подростков был выявлен избыток жировой массы при нормальном значении SDS ИMT $(+0,22 ;+0,36)$, у остальных пациентов значения жировой и тощей массы находились в пределах референса. Во 2-й группе пациентов без андрогенного дефицита $(n=17)$ нормальные показатели композиционного состава тела определились в 70,6\% случаев. Дефицит жировой ткани выявлен у двоих подростков. Избыток жировой массы определен у 4 подростков: у 1 пациента с нормальным SDS ИMT $(-0,17)$, у 1 пациента с избытком веса (SDS ИMТ: $+1,9)$ и у 2 подростков с ожирением (SDS ИMТ: $+2,3 ;+2)$. Таким образом, среди 6 пациентов $(21,4 \%)$, у которых был выявлен избыток жировой ткани по данным биоимпедансного анализа, половина имела нормальный SDS ИMT.

По результатам оценки ОО более чем у половины подростков с СК (64,3\%) выявлены нормальные значения энергетического обмена в покое (рис. 1). В группе

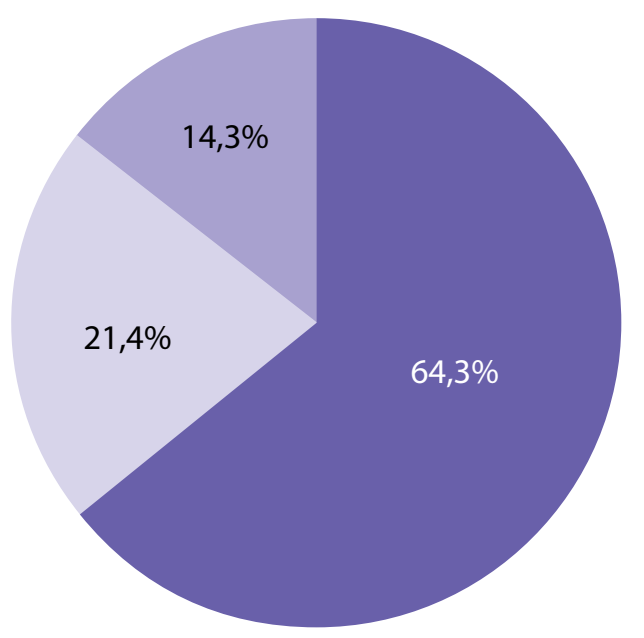

Норма $(n=18)$

Сниженный ОО (n=6)

Повышенный ОО (n=4)

Рисунок 1. Анализ значения основного обмена в общей группе пациентов с синдромом Клайнфельтера. с андрогенным дефицитом снижение интенсивности ОО было определено лишь у 1 подростка (9\%), нормальные показатели регистрировались в $82 \%$ случаев. Во 2-й группе снижение скорости ОО было выявлено у 4 пациентов (21\%), 2 из которых имели ожирение. Увеличение скорости ОО было определено у 3 подростков с СК с нормальными значениями SDS ИМТ и параметрами композиционного состава тела.

При проведении корреляционного анализа выявлена положительная взаимосвязь ОО с количеством тощей массы $(r=0,55, p<0,05)$ и SDS ИМТ $(r=0,33, p<0,05)$, но не с процентным содержанием жировой массы в организме $(r=0,09, p<0,05)$.

При оценке влияния андрогенного статуса на состояние ОО в исследуемых группах продемонстрировано, что показатели общего тестостерона значимо различались (8,7 vs 11,4 нмоль/л) при сопоставимых абсолютных значениях энергозатрат в покое, а также с поправкой на тощую массу (табл. 2, рис. 2).

Корреляционный анализ ОО с поправкой на тощую массу и общего тестостерона не выявил взаимосвязи между этими показателями в группе пациентов, имеющих

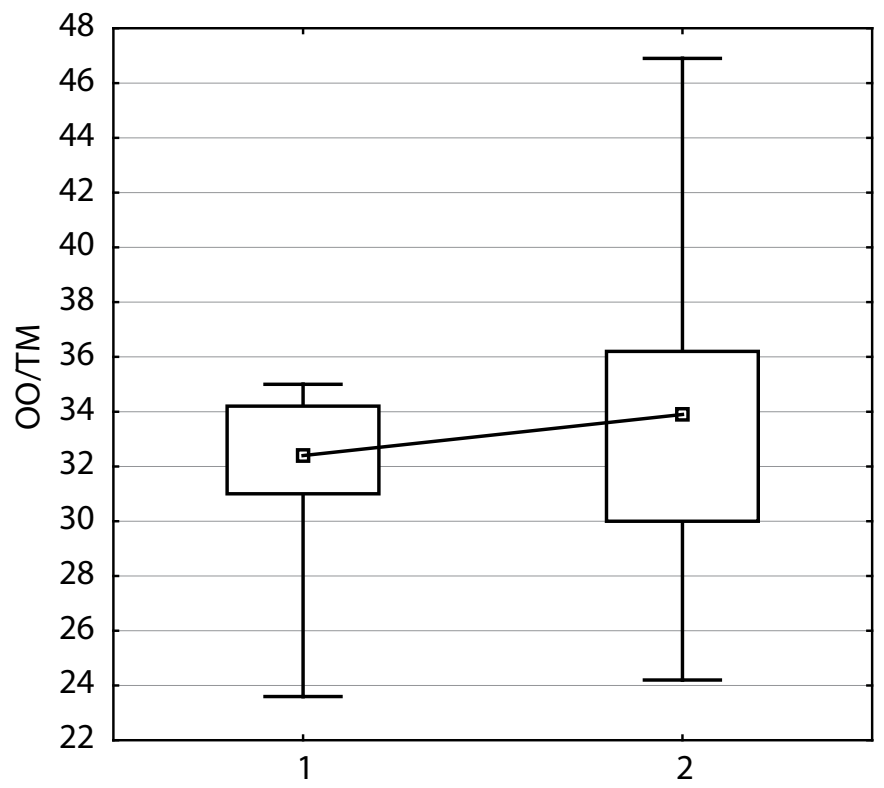

Группы

* Медиана $\quad$ 25-75\% I Размах без выбр.

Рисунок 2. Сравнительный анализ 2 групп пациентов по показателю $\mathrm{OO} / \mathrm{TM}(\mathrm{p}=0,36)$. ОО - основной обмен; ТM - тощая масса.

Таблица 2. Результаты анализа композиционного состава тела и основного обмена в обеих группах

\begin{tabular}{lccc}
\hline \multicolumn{1}{c}{ Группа } & $\mathbf{1}$ & $\mathbf{2}$ & $\mathbf{p}$ \\
\hline $\mathrm{n}$ & 11 & 17 & 0,37 \\
Жировая ткань, кг & $11,3[8,4 ; 13,5]$ & $9,8[7,4 ; 11,8]$ & 0,87 \\
Содержание жира, \% & $16,3[14,9 ; 20,1]$ & $17,0[13,7 ; 19,4]$ & 0,42 \\
Тощая масса, кг & $51,0[47,7 ; 57,3$ & $50,4[44,8 ; 54,7]$ & 0,42 \\
Водный коэффициент, кг & $37,3[34,9 ; 41,9]$ & $36,9[32,8 ; 40,0]$ & 0,93 \\
ОО, ккал/сут & $1609[1500 ; 1938]$ & $1653[1520 ; 1770]$ & 0,36 \\
ОО/ТМ, ккал/сут/кг & $32,4[31,0 ; 34,2]$ & $33,9[30,0 ; 36,2]$ & \\
\hline
\end{tabular}

Примечание. Данные представлены в виде: Ме $[\mathrm{QH} ; \mathrm{QB}]$. Уровень статистической значимости различий принимался при р<0,05. 


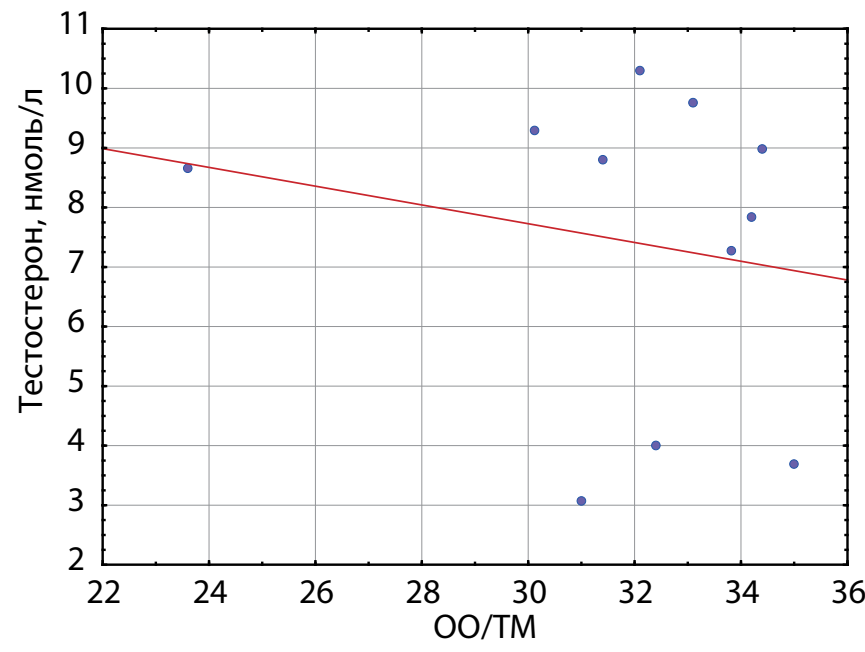

Рисунок. 3. Корреляция показателя ОО/ТМ с уровнем тестостерона у пациентов 1 группы (с признаками андрогенного дефицита) $(r=-0,17 ; p<0,05)$. ОО - основной обмен; ТM - тощая масса.

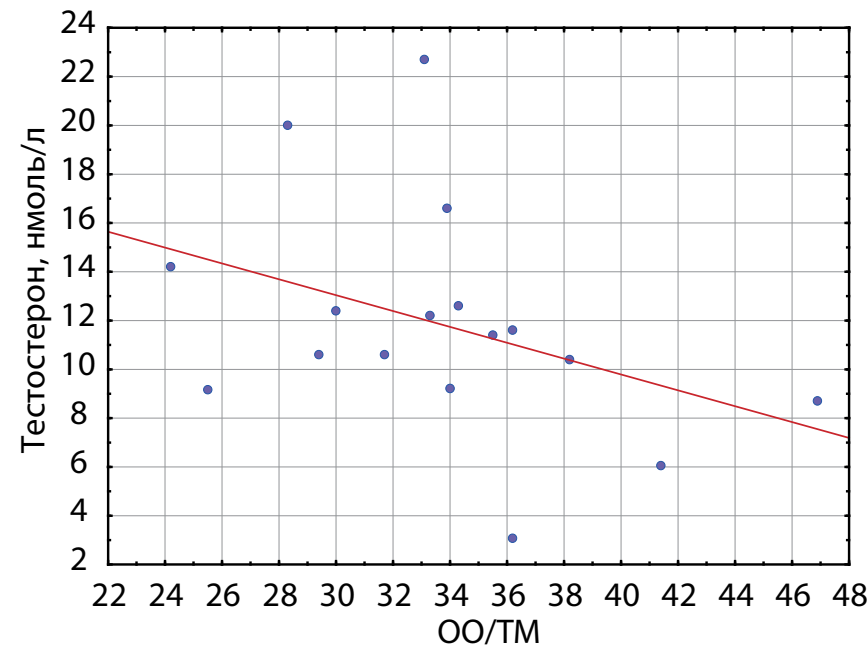

Рисунок. 4. Корреляция показателя ОО/ТМ с уровнем тестостерона у пациентов 2 группы (без признаков андрогенного дефицита) $(r=-0,51 ; p<0,05)$. ОО - основной обмен; TM - тощая масса.

\section{ОБСУЖДЕНИЕ}

признаки гипогонадизма $(r=-0,17, p<0,05)$ (рис. 3). Однако в группе без признаков гипогонадизма продемонстрирована отрицательная корреляционная связь ОО/ТМ с тестостероном сыворотки ( $r=-0,51, p<0,05)$ (рис. 4).

При оценке частоты метаболических нарушений у подростков с СК ИР была выявлена в 39,3\% случаев (11 пациентов). При сравнительном анализе обеих групп по индексу НОМА не выявлено статистически значимых различий $(\mathrm{p}=0,42)$. Нарушение липидного обмена зарегистрировано лишь у одного подростка (3,6\%). Нарушений углеводного обмена по данным ОГТТ у подростков с СК выявлено не было. По уровню гликемии натощак, уровням общего холестерина, триглицеридов, ЛПНП, АЛТ, АСТ исследуемые группы значимо не различались (табл. 3). При проведении корреляционного анализа выявлена взаимосвязь уровня ИРИ натощак с количеством тощей массы $(r=0,43$, $\mathrm{p}<0,05)$. Не найдено ассоциаций между процентным содержанием жировой массы в организме с частотой дислипидемии, ИР или нарушений углеводного обмена.

Нежелательные явления

В ходе исследования нежелательных явлений зафиксировано не было.
Резюме основного результата исследования

Для большинства подростков с СК, независимо от наличия клинических и лабораторных признаков гипогонадизма, характерны нормальные показатели композиционного состава тела, основного обмена и метаболического профиля.

Показатели композиционного состава тела и интенсивность энергетического обмена не зависят от уровня общего тестостерона в сыворотке крови.

\section{Обсуждение основного результата исследования}

Анализ научных публикаций последних лет продемонстрировал актуальность изучения возможных механизмов развития метаболических нарушений и ассоциированных заболеваний у пациентов с СК. У взрослых пациентов с СК значительно чаще, чем в популяции, выявляется ожирение (в 50\% случаев), в том числе абдоминальное [2]. У детей и подростков с СК, по данным исследования Pacenza и соавт., включившего 44 пациента, ожирение было выявлено в 15,9\% случаев [11]. В нашем исследовании частота ожирения

Таблица 3. Результаты анализа метаболических показателей крови в обеих группах

\begin{tabular}{lccc}
\hline & Группа & $\mathbf{1}$ & $\mathbf{p}$ \\
\hline $\mathrm{n}$ & 11 & 17 & 0,22 \\
ТГ, ммоль/л & $0,9[0,64 ; 1,21]$ & $0,74[0,57 ; 0,87]$ & 0,67 \\
Общий ХС, ммоль/л & $3,94[3,41 ; 4,47]$ & $4,04[3,72 ; 4,58]$ & 0,42 \\
ЛПНП, ммоль/л & $2,32[2,14 ; 2,67]$ & $2,65[2,08 ; 3,15]$ & 0,03 \\
ЛПВП, ммоль/л & $1,25[1,19 ; 1,36]$ & $1,14[1,05 ; 1,23]$ & 0,64 \\
АЛТ, Ед/л & $14[12 ; 17]$ & $12[10 ; 14]$ & 0,06 \\
АСТ, Ед/л & $19[18 ; 20]$ & $17,0[16 ; 18]$ & 0,19 \\
Глюкоза, ммоль/л & $4,68[4,2 ; 4,98]$ & $4,43[4,31 ; 4,97]$ & 0,72 \\
ИРИ, мкЕ/мл & $15,6[13,39 ; 19,18]$ & $14,2[8,78 ; 21,85]$ & 0,42 \\
НОМА & $3,15[2,55 ; 3,56]$ & $2,07[1,65 ; 4,4]$ & \\
\hline
\end{tabular}

Примечание. Данные представлены в виде: Ме [QH; QB]. Уровень статистической значимости различий принимался при р<0,05. 
и избыточной массы тела у подростков с СК составила $10,7 \%$.

Дефицит андрогенов у мужчин способствует избыточному накоплению жировой ткани и ассоциирован с дефицитом мышечной массы, а также снижением мышечной силы [12]. В ряде исследований, оценивавших эффект заместительной терапии препаратами тестостерона у мужчин с гипогонадизмом различной этиологии, было отмечено благоприятное влияние терапии на углеводный и липидный обмен, композиционный состав тела (в связи с увеличением количества тощей массы и снижением количества жировой ткани), а также на особенности топографии жировой ткани, характеризующиеся уменьшением количества висцерального жира $[13,14]$.

В проведенном нами исследовании у 71,4\% подростков с СК отмечались нормальные показатели композиционного состава тела. В 24\% случаев был выявлен избыток жировой ткани, причем у 10,7\% подростков - с нормальным SDS ИMT. Таким образом, у части подростков с СК отмечается увеличение содержания жировой ткани в организме даже при отсутствии ожирения или избыточной массы тела. В нашем исследовании оценка композиционного состава тела проводилась методом биоимпедансного анализа, что не позволило объективно оценить висцеральное жировое депо у подростков с СК.

В ряде работ, оценивавших процентное содержание жировой ткани у детей и подростков с СК, продемонстрировано, что избыточное отложение жировой ткани, в особенности в абдоминальной области, может наблюдаться еще до развития гипогонадизма и даже в отсутствие ожирения [3, 4]. В исследовании Aksglaede и соавт., включившем 24 ребенка с СК, было показано отсутствие значимых различий в содержании мышечной и жировой массы между группой подростков, получавших заместительную терапию андрогенами, и группой не получавших лечения [3]. В нашем исследовании получены схожие результаты, которые продемонстрировали отсутствие влияния дефицита андрогенов на композиционный состав тела. Выявленная отрицательная корреляционная связь между уровнем тестостерона и показателем ОО/ТМ в группе без гипогонадизма (см. рис. 4) объясняется тем, что андрогены оказывают свой анаболический эффект не на всю тощую массу, а лишь на скелетную мускулатуру, которая в покое является метаболически неактивной.

Анализ данных литературы о влиянии терапии препаратами тестостерона на композиционный состав тела у взрослых пациентов с СК показал противоречивые результаты: есть сообщения о частичном положительном эффекте [15] и об отсутствии каких-либо значимых изменений на фоне лечения [16]. В недавнем плацебо-контролируемом исследовании с участием 13 взрослых мужчин с СК в возрасте от 21 года до 53 лет было продемонстрировано уменьшение количества висцерального жира (оценен по данным компьютерной томографии) и общего содержания жировой ткани в организме, а также увеличение массы мышечной ткани в группе пациентов, в течение 6 месяцев получавших заместительную терапию препаратами тестостерона, по сравнению с группой плацебо [17].

Снижение ОО может способствовать формированию «положительного» энергетического баланса и набору веса у лиц с ожирением [9]. Проводя анализ литературы о предполагаемых механизмах развития ожирения при
СК, мы не нашли работ, посвященных оценке ОО у данной группы пациентов. В нашем исследовании более чем у половины подростков с СК выявлен нормальный уровень ОО, а его снижение отмечается в 21,4\% случаев и не связано с более высоким SDS ИМТ. Как и при конституционально-экзогенном ожирении, при СК тощая масса вносит основной вклад в формирование энергообмена в покое, что косвенно подтверждает выявленная нами корреляционная взаимосвязь абсолютных значений ОО с количеством тощей, но не жировой массы. Анализ в группах в зависимости от наличия признаков гипогонадизма не выявил существенных различий в скорости ОО, в том числе после внесения поправки на тощую массу (см. рис. 2), что указывает на отсутствие непосредственного влияния дефицита андрогенов на интенсивность энергетического обмена в покое.

Наиболее частым метаболическим нарушением у подростков с СК, по нашим данным, стала ИР, выявленная в 39,3\% случаев. Подобные результаты продемонстрированы и другими авторами [18]. Снижение чувствительности к инсулину у подростков с СК, в том числе, может быть обусловлено особенностями топографии жировой ткани с ее преимущественным висцеральным перераспределением. Дислипидемия в нашем исследовании определена лишь в 3,6\% случаев. В работе Bardsley и соавт. с участием 89 пациентов с СК допубертатного возраста (от 4 до 12,9 года) частота дислипидемии составила 37\%, что оказалось значительно выше по сравнению с показанной нами [18]. Данных литературы о частоте нарушения толерантности к глюкозе у детей и подростков с СК нами найдено не было.

Наиболее вероятной причиной низкой частоты метаболических нарушений при СК, продемонстрированной в нашем исследовании, является малое количество пациентов с избыточной массой тела и ожирением.

Учитывая неоднозначность влияния андрогенного дефицита и композиционного состава тела на развитие метаболических нарушений у пациентов с СК, в последнее время значительную роль в их формировании отводят пока что малоизученным эффектам дополнительной Х-хромосомы. Известно, что, несмотря на наличие механизма инактивации второй (у здоровых женщин) и каждой дополнительной X-хромосомы (при СК), обеспечивающего дозовую компенсацию генов, примерно 15\% генов инактивации не подвергаются, приводя к их гиперэкспрессии [19]. Выявлена взаимосвязь гиперэкспрессии гена CSF2RA, кодирующего белок-рецептор семейства цитокинов, с наличием ИР, абдоминального ожирения и повышения концентрации ингибитора активатора плазминогена 1 типа (PAl-1) в сыворотке у пациентов с СК [20].

Принимая во внимание небольшое число исследований, изучавших влияние эпигенетических факторов на развитие ассоциированных заболеваний у пациентов с СК, вопрос этиопатогенеза метаболических нарушений остается открытым и требует проведения новых исследований с участием больших групп пациентов.

\section{Ограничения исследования}

Биоимпедансный анализ состава тела не позволяет объективно оценить количество висцерального жира в организме, в связи с чем в данной работе проведена 
оценка особенности топографии жировой ткани и влияния висцерального ожирения на развитие метаболических нарушений. Также данный метод не позволяет дифференцировать скелетную мышечную массу от массы внутренних органов, вносящую основной вклад в формирование ОО.

\section{ЗАКЛЮЧЕНИЕ}

Показатели композиционного состава тела и ОО у подростков с СК в нашем исследовании не ассоциированы с уровнем общего тестостерона в сыворотке крови, что свидетельствует о незначительном влиянии андрогенного дефицита на данные параметры.

У подростков с СК отмечается низкая частота ожирения и избыточной массы тела, однако у части пациентов с нормальным SDS ИMT выявляется избыточное количество жировой ткани.
Для подростков с СК без ожирения характерна низкая частота метаболических нарушений.

\section{ДОПОЛНИТЕЛЬНАЯ ИНФОРМАЦИЯ}

Источник финансирования. Фонд поддержки и развития филантропии «КАФ» в рамках благотворительной программы «Альфа-Эндо».

Конфликт интересов. Авторы декларируют отсутствие явных и потенциальных конфликтов интересов, связанных с публикацией настоящей статьи, о которых необходимо сообщить.

Участие авторов. Концепция и дизайн исследования Беспалюк Д.А., Окороков П.Л., Чугунов И.С.; проведение биоимпедансометрии и непрямой респираторной калориметрии - Окороков П.Л.; написание текста - Беспалюк Д.А.; статистическая обработка данных Беспалюк Д.А.; редакция текста, внесение ценных замечаний Окороков П.Л., Чугунов И.С. Все авторы внесли существенный вклад в проведение исследования и подготовку статьи, прочли и одобрили финальную версию перед публикацией.

\section{СПИСОК ЛИТЕРАТУРЫ | REFERENCES}

1. Salzano A, D'Assante R, Heaney LM, et al. Klinefelter syndrome, insulin resistance, metabolic syndrome, and diabetes: review of literature and clinical perspective. Endocrine. 2018;61(2):194-203. doi: 10.1007/s12020-018-1584-6.

2. Groth KA, Skakkebæk A, Høst C, et al. Clinical review: Klinefelter syndrome - a clinical update. J Clin Endocrinol Metab. 2013;98(1):20-30. doi: 10.1210/jc.2012-2382

3. Aksglaede L, Molgaard C, Skakkebaek NE, et al. Normal bone mineral content but unfavourable muscle/fat ratio in Klinefelter syndrome. Arch Dis Child. 2008;93(1):30-34. doi: 10.1136/adc.2007.120675.

4. Davis S, Lahlou N, Bardsley M, et al. Gonadal function is associated with cardiometabolic health in pre-pubertal boys with Klinefelter syndrome. Andrology. 2016;4(6):1169-1177. doi: 10.1111/andr.12275.

5. Bojesen A, Kristensen K, Birkebaek NH, et al. The metabolic syndrome is frequent in Klinefelter's syndrome and is associated with abdominal obesity and hypogonadism. Diabetes Care. 2006;29(7):1591-1598. doi: 10.2337/dc06-0145.

6. Yesilova Z, Oktenli C, Sanisoglu SY, et al. Evaluation of insulin sensitivity in patients with Klinefelter's syndrome: a hyperinsulinemic euglycemic clamp study. Endocrine. 2005;27(1):11-15. doi: 10.1385/ENDO:27:1:011.

7. Dimopoulou C, Goulis DG, Corona G, et al. The complex association between metabolic syndrome and male hypogonadism. Metabolism. 2018;86:61-68. doi: 10.1016/j.metabol.2018.03.024.

8. Bosy-Westphal A, Kossel E, Goele K, et al. Contribution of individual organ mass loss to weight loss-associated decline in resting energy expenditure. Am J Clin Nutr. 2009;90(4):993-1001. doi: 10.3945/ajen.2008.27402.

9. Окороков П.Л., Васюкова О.В., Ширяева Т.Ю. Скорость основного обмена в покое и факторы его вариабельности у подростков с простым ожирением // Вопросы детской диетологии. 2019. - T.17. - №3. - C. 5-9. [Okorokov PL, Vasyukova OV, Shiryaeva TYu. Resting metabolic rate and factors of its variability in adolescents with obesity. Voprosy detskoy diyetologii. 2019;17(3):5-9. (In Russ).] doi: 10.20953/1727-5784-2019-3-5-9.

10. McCarthy HD, Cole TJ, Fry T, et al. Body fat reference curves for children. Int J Obes (Lond). 2006;30(4):598-602. doi: 10.1038/sj.ijo.0803232

11. Pacenza N, Pasqualini T, Gottlieb S, et al. Clinical presentation of Klinefelter's syndrome: differences according to age. Int J Endocrinol. 2012;324835. doi: 10.1155/2012/324835.
12. Roy TA, Blackman MR, Harman SM, et al. Interrelationships of serum testosterone and free testosterone index with FFM and strength in aging men. Am J Physiol Endocrinol Metab. 2002;283(2):E284-E294. doi: 10.1152/ajpendo.00334.2001.

13. Srinivas-Shankar U, Roberts SA, Connolly MJ, et al. Effects of testosterone on muscle strength, physical function, body composition, and quality of life in intermediate-frail and frail elderly men: a randomized, double-blind, placebo-controlled study. J Clin Endocrinol Metab. 2010;95(2):639-650. doi: 10.1210/jc.2009-1251.

14. Тишова Ю.А., Калинченко С.Ю. Роль коррекции гипогонадизма в лечении метаболического синдрома у мужчин и аспекты безопасности терапии препаратами тестостерона пролонгированного действия (результаты двойного слепого рандомизированного плацебо-контролируемого исследования) // Ожирение и метаболизм. - 2010. - Т.7. №2. - C. 36-43. [Tishova YuA, Kalinchenko SYu. Rol' korrektsii gipogonadizma v lechenii metabolicheskogo sindroma u muzhchin i aspekty bezopasnosti terapii preparatami testosterona prolongirovannogo deystviya (rezul'taty dvoynogo slepogo randomizirovannogo platsebo-kontroliruyemogo issledovaniya). Obesity and metabolism. 2010;7(2):36-43. (In Russ).]

15. Chang S, Skakkebæk A, Trolle C, et al. Anthropometry in Klinefelter syndrome - multifactorial influences due to CAG length, testosterone treatment and possibly intrauterine hypogonadism. J Clin Endocrinol Metab. 2015;100(3):508-517. doi: 10.1210/jc.2014-2834.

16. Jo DG, Lee HS, Joo YM, et al. Effect of testosterone replacement therapy on bone mineral density in patients with Klinefelter syndrome. Yonsei Med J. 2013;54(6):1331-1335. doi: 10.3349/ymj.2013.54.6.1331.

17. Høst C, Bojesen A, Erlandsen M, et al. A placebo-controlled randomized study with testosterone in Klinefelter syndrome beneficial effects on body composition. Endocr Connect. 2019;8(9):1250-1261. doi: 10.1530/EC-19-0323.

18. Bardsley $\mathrm{MZ}$, Falkner B, Kowal K, et al. Insulin resistance and metabolic syndrome in prepubertal boys with Klinefelter syndrome. Acta Paediatr. 2011;100(6):866-870. doi: 10.1111/j.1651-2227.2011.02161.x.

19. Berletch JB, Yang $F, X u J$, et al. Genes that escape from $X$ inactivation Hum Genet. 2011;130(2):237-245. doi: 10.1007/s00439-011-1011-z.

20. Zitzmann M, Bongers R, Werler S, et al. Gene expression patterns in relation to the clinical phenotype in Klinefelter syndrome. J Clin Endocrinol Metab. 2015;100(3):518-523. doi: 10.1210/jc.2014-2780.

Рукопись получена: 20.03.2020. Одобрена к публикации: 17.04.2020. Опубликована online: 25.07.2020. 


\section{ИНФОРМАЦИЯ ОБ АВТОРАХ [AUTHORS INFO]}

*Беспалюк Дарья Алексеевна [Daria A. Bespalyuk, MD]; адрес: Россия, 117036, Москва, ул. Дм. Ульянова, д. 11 [address: 11 Dm.Ulyanova street, Moscow, 117036, Russia]; ORCID: https://orcid.org/0000-0002-4900-6652; eLibrary SPIN: 7129-8794; e-mail: dariabespalyuk@gmail.com

Окороков Павел Леонидович, к.M.н. [Pavel L. Okorokov, MD, PhD]; ORCID: http://orcid.org/0000-0001-9834-727X; eLibrary SPIN: 6989-2620; e-mail: pokorokov@gmail.com

Чугунов Игорь Сергеевич, к.м.н. [Igor S.Chugunov, MD, PhD]; ORCID: https://orcid.org/0000-0003-4915-1267;

eLibrary SPIN: 1514-5005; e-mail: chugunovigor@gmail.com

\section{ЦИТИРОВАТЬ:}

Беспалюк Д.А., Окороков П.Л., Чугунов И.С. Анализ композиционного состава тела, основного обмена и частоты метаболических нарушений у подростков с синдромом Клайнфельтера // Проблемы эндокринологии. - 2020. — Т.66. №2. - C. 71-78. doi: https://doi.org/10.14341/probl12360

\section{FOR CITATION:}

Bespalyuk DA, Okorokov PL, Chugunov IS. Evaluation of body composition, resting metabolic rate and frequency of metabolic disorders in adolescents with Klinefelter syndrome. Problems of Endocrinology. 2020;66(2):71-78. doi: https://doi.org/10.14341/probl12360 